\title{
Editorial
}

\section{CONTRIBUCIONES AL DESARROLLO DE LA INVESTIGACIÓN EN ENFERMERÍA: RETOS Y PERSPECTIVAS}

\author{
CONTRIBUTIONS TO THE DEVELOPMENT OF NURSING RESEARCH: CHALLENGES \\ AND PERSPECTIVES \\ Myriam Durán Parra ${ }^{1}$, Wilson Cañón Montañez $z^{2}$, Alexandra Marieth Barajas Blanco ${ }^{3}$
}

Desde la etimología del término "investigación” la palabra proviene del latín in (en) y vestigare (hallar, inquirir, indagar, seguir vestigios). De ahí el uso más elemental del término en el sentido de "averiguar o describir alguna cosa". Desde el momento en que el hombre se enfrentó a problemas y frente a ellos empezó a interrogarse sobre el porqué, como y para que, con esta indagación sobre las cosas, de una manera embrionaria, comenzó lo que hoy llamamos investigación. Con el transcurso del tiempo el ser humano ha hecho un esfuerzo importante por sobrevivir y la mejor forma de lograrlo ha sido experimentando aportando al desarrollo del conocimiento científico.

Donaldson y Bottorff (1978) expresan frente a la investigación en enfermería que "es la fuente de desarrollo de conocimiento que le da la característica de disciplina, que ha de estar presente tanto en la práctica clínica como en el desarrollo de la administración y la educación, que ha de dar respuesta a las necesidades sociales que están relacionadas con procesos de salud-enfermedad, condiciones y calidad de vida de las personas, familias y comunidades, en todos los grupos etarios" (1). Por su parte Borrero-Cabal (1994), afirma que "la disciplina conlleva al sentido de rigor, de dedicación, de entrenamiento y ejercicio de los hábitos científicos de la persona para elaborar, transmitir y aprender una ciencia” (2). En enfermería esto se traduce que para poder llevar el cuidado a los pacientes de una manera técnica, científica, analítica, debe tener unas bases como la dedicación, el amor por la profesión y el gusto por la investigación reconociendo que la enfermería avanza y se fortalece a medida que crece científicamente, mientras conoce la cultura, la riqueza de la naturaleza, forma parte del país y sobre todo del mundo como una profesión que demuestra su interés en la búsqueda de su propia identidad.

Si recordamos a Florence Nightingale (1820-1910) ha sido objeto de estudio como "reformadora, como estadística, como investigadora; tras la guerra de Crimea (1854), Nightingale escribió unos doscientos libros, informes y opúsculos que tuvieron importantes repercusiones en la sanidad militar, la asistencia social en la India, los hospitales civiles, las estadísticas médicas y la asistencia a los enfermos" (3). Esto es una muestra convincente de que la enfermería desde hace muchos años ha trazado sus límites fortaleciéndose en la investigación como una disciplina que para la práctica del cuidado tiene un abordaje científico e investigativo que la soporta, lo cual ha permitido enriquecer el acerbo de una ciencia dirigida a crear un cuerpo de conocimientos organizados y aportar al desarrollo de los países.

Hoy ante la aceleración del conocimiento y la práctica en el cuidado de enfermería en el mundo moderno, las necesidades actuales en materia de investigación son diversas y requieren una constante actualización. La Asociación Colombiana de Facultades de Enfermería (ACOFAEN), comprometida con el desarrollo de la investigación, encamina acciones para el fortalecimiento profesional en el marco de la excelencia en la calidad de los procesos de formación y que respondan a la resolución de problemas de la sociedad colombiana. En este sentido ACOFAEN, se ha propuesto como meta impactar en las unidades académicas, en el componente de investigación, "mediante la creación del Comité Permanente de Investigación (COPEI), que se constituye en el órgano consultivo y asesor en investigación de enfermería, el cual como su denominación lo indica, monitorea de manera ágil y permanente el desarrollo del conocimiento enfermero en el país desde la perspectiva de la evidencia científica, al igual que recepciona los aportes de los diferentes grupos y líneas de investigación existentes en enfermería para contribuir al desarrollo de la disciplina” (4).

La academia, la empresa, el gobierno son claves para generar conocimiento, considerando la primera quizás uno de los actores más importantes en tanto que es el escenario de la formación y la producción del recurso humano; enfrentándose claro, a retos enormes por cuanto debe lograr romper la brecha existente entre el ámbito de servicio y el de la formación.

ACOFAEN, como entidad académica científica ha contribuido positivamente, con resultados de investigaciones financiadas con diversos organismos nacionales e internacionales, al progreso de grupos poblacionales en el país, en donde han participado un gran

${ }^{1}$ Enfermera. Magíster en Enfermería con Énfasis en Gerencia de Servicios de Salud. Presidenta Asociación Colombiana de Facultades de Enfermería ACOFAEN. Directora Revista CUIDARTE. Directora Programa de Enfermería. Líder Grupo de Investigación de Enfermería- Everest. Universidad de Santander UDES.

${ }^{2}$ Enfermero. Magíster en Epidemiologia. Doctorando en Epidemiologia. Editor en Jefe Revista CUIDARTE, Profesor, Programa de Enfermería, Universidad de Santander UDES. 
número de investigadores de enfermería de todo el país. De esta manera se organizan eventos de índole nacional e internacional que permiten espacios de reflexión e integración para afianzar y articular esfuerzos en el fin común de la visibilización y crecimiento de la ciencia de Enfermería; es así como en el 2014, Colombia será sede del XIV Coloquio Panamericano de Investigación en Enfermería.

El papel que cumple la educación superior en medio de esta revolución científica tiene que ver con el desarrollo de políticas que son esenciales para obtener mayor ventaja en la competencia internacional, por ello tiene la responsabilidad de continuar consolidando a la comunidad académica; fomentando la investigación y creando espacios que permitan el desarrollo de capacidades de trabajo en equipo; comunicación y sensibilidad social, responsabilidades con actitud crítica para resolver problemas de manera conjunta, con redes de académicos universitarios y de movilidad entre docentes y estudiantes y se puedan considerar proyectos de investigación conjunta que atiendan a las necesidades reales del país, de tal forma que se genere y aplique el conocimiento. En Colombia a través del Departamento Administrativo de Ciencia, Tecnología e Innovación (Colciencias) se hacen importantes acciones para consolidar la investigación y apoyar financieramente proyectos y cualificación del talento humano para la formación de científicos de excelencia, sin embargo, se requiere de mayor apoyo así como de mejorar los reconocimientos económicos y sociales.

La Revista de Investigación “CUIDARTE” del Programa de Enfermería de la Universidad de Santander UDES, tiene como filosofía servir de medio de divulgación de los trabajos producto de investigación de los profesionales de enfermería en aras de contribuir al avance investigativo de la disciplina.

Para ilustrar el origen del nombre de la Revista CUIDARTE, vale la pena mencionar que el arte es aquella expresión externa de las capacidades, emociones, aptitudes internas que se transmiten en pintar, crear, observar aquellas formas de expresión. Según Zamuner (2009) "Si hubiese que definir en forma sencilla y expresiva la profesión de enfermería, se podría definir en las siguientes palabras: es la ciencia y el arte del cuidado, ciencia porque implica una serie de conocimientos científicos de diversas disciplinas; y arte, porque mas allá de los saberes intelectuales requiere cierta cuota de intuición creativa para integrar lo cognitivo a la necesidad de atención" (5).

La Revista CUIDARTE, es una publicación en español y actualmente publica resultados de investigación en el ámbito nacional e internacional. Para la Edición 2012, se presentan novedades y por primera vez se publican artículos en portugués de colegas, investigadoras y profesionales de enfermería del Brasil. Esto es una gran oportunidad, dado que a pesar de las barreras del idioma, la enfermería y el arte de cuidar es uno solo, y es muy importante conocer y compartir las experiencias de investigación de los profesionales de enfermería de todo el mundo. Asimismo, se convierte en un desafío para que en las futuras ediciones de la revista se publiquen artículos en los tres importantes idiomas: inglés, español y portugués.

Por último, y hablando del "arte de cuidar", citamos las palabras de Florence Nightingale: "la enfermería es un arte y si se pretende que sea un arte que requiere una devoción tan exclusiva, una preparación tan dura, como el trabajo de un pintor o de un escultor, pero ¿cómo puede compararse la tela muerte o el frío mármol con el tener que trabajar con el cuerpo vivo, el templo del espíritu de Dios? Es una de las bellas artes; casi diría, la más bella de las bellas artes" (6).

\section{REFERENCIAS BIBLIOGRÁFICAS}

1. Donaldson SK, Crowley DM. The discipline of nursing. Nurs Outlook. 1978 Feb;26(2):113-20.

2. Borrero-Cabal A. La interdisciplinariedad. En: Política y gestión universitaria. Cali: Universidad del Valle; 1994. p.18.

3. Attewell A. Florence Nightingale (1820-1910). Perspectivas: Revista Trimestral de Educación Comparada. 1988. 28(1): 173-189.

4. Comité Permanente de Investigación (COPEI). Asociación Colombiana de Facultades de Enfermería (ACOFAEN). Documento COPEI 2012, pag 3.

5. Zamuner M. La enfermería, ciencia y arte del cuidado. Revista Temas de Enfermería Actualizados TEA; 2009: 1-3.

6. Narváez-Traverso A, Martínez-Galiano JM, Pérez-Martín B. Revisitando a Florence Nightingale desde una perspectiva de género. Index Enferm [revista en línea]. 2010 [Consultado 1 Noviembre de 2012]; 19(4): 299302. Disponible en : http://scielo.isciii.es/scielo.php?pid=S1132-12962010000300014\&script=sci_arttext 